\title{
Frontal cerebral oxygenation asymmetry: intersubject variability and dependence on systemic physiology, season, and time of day
}

\author{
Hamoon Zohdi, ${ }^{\text {a }}$ Felix Scholkmann, ${ }^{\text {a,b, }, \uparrow}$ and Ursula Wolf ${ }^{\mathrm{a},}, * *$ \\ ${ }^{a}$ University of Bern, Institute of Complementary and Integrative Medicine, Bern, Switzerland \\ ${ }^{b}$ University of Zurich, University Hospital Zurich, Biomedical Optics Research Laboratory, \\ Department of Neonatology, Zurich, Switzerland
}

\begin{abstract}
Significance: Our study reveals that frontal cerebral oxygenation asymmetry (FCOA), i.e. a difference in the oxygenation between the right and left prefrontal cortex (PFC), is a real phenomenon in healthy human subjects at rest.

Aim: To investigate FCOA, we performed a study with 134 healthy right-handed subjects with the systemic physiology augmented functional near infrared spectroscopy (SPA-fNIRS) approach.

Approach: Subjects were measured 2 to 4 times on different days resulting in an unprecedented number of 518 single measurements of the absolute values of tissue oxygen saturation $\left(\mathrm{StO}_{2}\right)$ and total hemoglobin concentration ([tHb]) of the right and left PFC. Measurements were performed with frequency-domain functional near-infrared spectroscopy. In addition, the cardiorespiratory parameters were measured simultaneously.

Results: We found that (i) subjects showed an FCOA (higher $\mathrm{StO}_{2}$ on the right PFC), but not for $\mathrm{tHb}$; (ii) intrasubject variability was excellent for both $\mathrm{StO}_{2}$ and $\mathrm{tHb}$, and fair for FCOA; (iii) $\mathrm{StO}_{2}$ correlated significantly with blood $\mathrm{CO}_{2}$ concentration, [tHb] with heart rate, respiration rate (RR), and the pulse-respiration quotient (PRQ), and FCOA with RR and PRQ; (iv) FCOA and $\mathrm{StO}_{2}$ were dependent on season and time of day, respectively; (v) FCOA was negatively correlated with the room temperature; and (vi) $\mathrm{StO}_{2}$ and tHb were not correlated with the subjects mood but with their chronotype, whereas FCOA was not dependent on the chronotype.

Conclusion: Our study demonstrates that FCOA is real, and it provides unique insights into this remarkable phenomenon.

(C) The Authors. Published by SPIE under a Creative Commons Attribution 4.0 Unported License. Distribution or reproduction of this work in whole or in part requires full attribution of the original publication, including its DOI. [DOI: 10.1117/1.NPh.7.2.025006]
\end{abstract}

Keywords: functional near-infrared spectroscopy; prefrontal cortex; right-left asymmetry; tissue oxygenation; systemic physiology.

Paper 20002R received Jan. 7, 2020; accepted for publication Jun. 8, 2020; published online Jun. 23, 2020 .

\section{Introduction}

Our own preliminary measurements suggested that cerebral oxygenation differed between the right and left prefrontal cortex (PFC) in healthy human adults at rest. ${ }^{1}$ This phenomenon, which we named frontal cerebral oxygenation asymmetry (FCOA), is characterized by higher tissue oxygenation over the right PFC compared to the left.

Hemispheric specialization has been reported for a wide range of cerebral functions. ${ }^{2}$ For example, it is known that the regions in the left hemisphere are usually dominant for language and logical processing, whereas regions in the right hemisphere are specialized for spatial recognition and emotional control. ${ }^{3,4}$ Lateralization of function was first reported in the domain of

*Address all correspondence to Ursula Wolf, E-mail: ursula.wolf@ikim.unibe.ch

${ }^{\dagger}$ These authors share last authorship. 
language functions, which is widely accepted as a fundamental feature of neural organization, where it was revealed that the left hemisphere is dominant in language processing. ${ }^{5,6}$ Additionally, more than $90 \%$ of the population prefers the right hand for manual activities, with superior fine motor control and motor strength, which is controlled by the left hemisphere. ${ }^{4}$ Research has demonstrated that neural organization exists for the control motor actions, where each brain hemisphere contributes exclusive control mechanisms to the movement of each arm. ${ }^{7}$ Mutha et al. ${ }^{7}$ suggested that the left hemisphere provides predictive control mechanisms, whereas the right one contributes positional control mechanisms during movement of either arm. In addition to lateralization of language and motor control, face processing has also been shown to have laterality to neural activity and connectivity. It has been demonstrated that areas in the right hemisphere are more anatomically connected, more synchronized during rest, and more actively communicating with each other during face perception compared to the left hemisphere. ${ }^{8}$ Interestingly, numerous electroencephalography (EEG) studies have demonstrated a right-left asymmetry in brain activity during the resting state. Frontal EEG asymmetry (FEA) activity has been explained using the approach-withdrawal model suggesting that there are two different types of motivation., ${ }^{9,10}$ The approach motivation signifies the propensity to move toward the desired stimulus and is associated with a higher left frontal activity, whereas the withdrawal/avoidance motivation indicates a propensity to move or stay away from an undesired stimulus and is associated with higher right frontal activity. ${ }^{11-13}$ However, only a few studies on the asymmetry of brain tissue oxygenation and metabolism have been performed so far. ${ }^{1,14}$ This prompted us to investigate this fascinating phenomenon in many subjects using functional near-infrared spectroscopy (fNIRS).

From a methodological point of view, three main types of NIRS-based optical tissue spectroscopy techniques have been developed so far: continuous wave (CW-NIRS), frequency domain (FD-NIRS), and time domain (TD-NIRS). CW-NIRS can provide information on concentration changes of oxyhemoglobin $\left(\left[\mathrm{O}_{2} \mathrm{Hb}\right]\right)$ and deoxyhemoglobin $([\mathrm{HHb}])$ but cannot determine absolute baseline values. Therefore, it is appropriate for applications in cognitive neuroscience as absolute values are not crucial and functional activity is relatively assessed with respect to the baseline. ${ }^{15}$ FD-NIRS and TD-NIRS measure not only the light intensity as CW NIRS but also the time of flight of photons through tissue. Therefore, time resolved techniques such as TD-NIRS and FD-NIRS are able to provide absolute $\left[\mathrm{O}_{2} \mathrm{Hb}\right],[\mathrm{HHb}]$, and total hemoglobin $([\mathrm{tHb}])$ concentrations as well as absolute tissue oxygen saturation $\left[\mathrm{StO}_{2}=\right.$ $\left.\left(\left[\mathrm{O}_{2} \mathrm{Hb}\right] /[\mathrm{tHb}]\right) \times 100\right] .{ }^{16,17}$ This is relevant additional information, e.g., the [tHb] is strictly proportional to cerebral blood volume by the hematocrit. Thus these systems have widely been used in many diverse fields and applications including clinical monitoring, traumatic brain injury, anesthesiology, neonatology, and psychiatry. ${ }^{18}$ A comprehensive review on the history of fNIRS development, methodology, and imaging instrumentation has been published by Scholkmann et al. ${ }^{19}$ In this study, we performed optical neuroimaging using multidistance FD-NIRS. This approach is also able to reduce the sensitivity to extracerebral tissue. It is known that the oxygenation of the brain depends on its activity state, and the metabolic changes in the brain are interrelated with systemic parameters. ${ }^{20-22}$ Therefore, it is essential to employ the systemic physiology augmented (SPA) fNIRS approach, which additionally and simultaneously measures absolute values of cardiorespiratory parameters including the end-tidal carbon dioxide $\left(\mathrm{P}_{\mathrm{ET}} \mathrm{CO}_{2}\right)$, heart rate $(\mathrm{HR})$, respiration rate (RR), and the pulse-respiration quotient (PRQ).

The main goal of this study was to investigate FCOA in a large number of healthy humans at rest to elucidate whether FCOA is a real and robust phenomenon. To facilitate a better understanding of this phenomenon, we employed SPA-fNIRS to assess whether FCOA depends on systemic physiological activity, absolute tissue oxygenation, or hemoglobin concentration. We also aimed to explore the effects of chronobiological and psychological variables on FCOA, as well as cerebral hemodynamics and oxygenation at the PFC during the resting state.

\section{Subjects and Methods}

\subsection{Subjects}

The study was carried out with 134 healthy subjects ( 85 female, 49 male, age $24.7 \pm 3.4$ years, and range 20 to 46 years). The subjects were all right-handed, according to the Edinburgh 
Handedness Inventory. ${ }^{23}$ Subjects were nonsmoking and indicated neither current nor previous history of neurological and psychiatric disorders or alcohol and drug abuse. Subjects were asked to refrain from consuming caffeine and eating $2 \mathrm{~h}$ prior to the experiment. The study protocol was approved by the Ethics Committee of the Canton of Bern. Informed consent was obtained from all subjects before the measurements. Subjects were also informed of their right to discontinue participation at any time.

\subsection{Experimental Protocol}

The resting state data were taken from a set of studies with different stimuli. Each measurement began with a baseline phase lasting $8 \mathrm{~min}$, during which the subjects sat upright in a comfortable chair in a dark room. For this study, we examined only the last $5 \mathrm{~min}$ of this baseline period. Each subject was measured on four different days but at the same time of day to prevent chronobiological artifacts. They were asked to keep their eyes open throughout the entire measurement and to move their head or body as little as possible during the measurement to avoid movement artifacts. Additionally, the subjects were asked to fill out two questionnaires before and after each measurement in order to assess their mood: the positive affect negative affect schedule $(\text { PANAS) })^{24}$ and the self-assessment manikin test (SAM; five points scale). ${ }^{25}$ The PANAS and SAM questionnaires are used as tools to measure state influence. Trait influence evaluation was not performed in our study. Additionally, we determined the chronotype by the Horne and Östberg morningness-eveningness questionnaire. ${ }^{26}$ Measurements were performed between 7:00 am and 9:00 pm. The mean room temperature was $22.8^{\circ} \mathrm{C} \pm 0.6^{\circ} \mathrm{C}$.

\subsection{Measurement Setup}

The Imagent (ISS Inc., Champaign, Illinois, USA), a multichannel FD-NIRS system, which employs a multidistance approach, was used to determine absolute values of the $\left[\mathrm{O}_{2} \mathrm{Hb}\right]$, [HHb], [tHb], and $\mathrm{StO}_{2}$ at a sampling rate of $2.5 \mathrm{~Hz}$ on the PFC. The Imagent's light source consists of 16 laser diodes at $760 \mathrm{~nm}$ and 16 laser diodes at $830 \mathrm{~nm}$. Four highly sensitive photomultiplier tubes serve as detectors. The sensors were placed bilaterally on the left and right prefrontal cortex (L-PFC and R-PFC) of subjects at position Fp1 and Fp2, according to the international 10 to 20 system. ${ }^{27}$ Each of the two ISS sensors had four light emitters and one light detector connected to an optical fiber delivering the light to the photomultiplier tube. The source-detector separations $(d)$ were $\sim 2.0,2.5,3.5$, and $4.0 \mathrm{~cm}$ with the sources and detectors arranged collinearly.

HR was measured by SOMNOtouch ${ }^{\mathrm{TM}}$ NIBP (SOMNOmedics GmbH, Randersacker, Germany) with a sampling rate of $4 \mathrm{~Hz}$. This device calculated the HR from the ECG data by calculating the $R-R$ intervals. RR and end-tidal carbon dioxide $\left(\mathrm{P}_{\mathrm{ET}} \mathrm{CO}_{2}\right)$ were measured noninvasively by a NONIN LifeSense (NONIN Medical, Plymouth, Minnesota, USA). Data were recorded at a sampling rate of $1 \mathrm{~Hz}$. All data were recorded simultaneously.

\subsection{Signal Processing and Statistical Analysis}

One subject was excluded from data analysis due to the perceived discomfort of the fNIRS sensors. 126 subjects completed all four measurements; only for seven subjects, the number of experimental sessions was lower. Therefore, the entire data for the current analysis comprised 518 single measurements. All signal processing was performed in MATLAB (R2017a, MathWorks, Inc., Massachusetts, USA).

\subsubsection{Cerebral oxygenation and hemodynamics}

Prior to analysis, data with extremely low $\left(\mathrm{StO}_{2}<40 \%\right)$ or improper high values $\left(\mathrm{StO}_{2}>100 \%\right)$ were removed by visual inspection. Movement artifacts in the $\mathrm{StO}_{2}$ and [tHb] signals were removed by the movement artifact reduction algorithm (MARA) based on moving standard deviation and piecewise-interpolation. ${ }^{28}$ For $86 \%$ of the signal time series, no processing with MARA was necessary. To remove high-frequency noise, signals were low pass filtered using a 
robust second-degree polynomial moving average (RLOESS) with a span of $2 \mathrm{~min}$. This method assigns zero weight to data outside six mean absolute deviations (MAD). For each measurement, the 5-min median of the baseline phase was calculated for each cerebral parameter. Absolute values of $\mathrm{StO}_{2}$ and $[\mathrm{tHb}]$ from the L-PFC and R-PFC were averaged to obtain a single value for the whole PFC. Moreover, the laterality index-defined as the difference between the absolute values for the R-PFC and L-PFC — was determined and is indicated by a " $\Delta$." Finally, median values and the interquartile range (IQR) of $\mathrm{StO}_{2}$ and $[\mathrm{tHb}]$ were calculated for each individual subject.

\subsubsection{Cardiorespiratory parameters}

All cardiorespiratory parameters, including $\mathrm{HR}, \mathrm{P}_{\mathrm{ET}} \mathrm{CO}_{2}$, and $\mathrm{RR}$, were also denoised by the RLOESS method with a window length of 3,1 , and $2 \mathrm{~min}$, respectively. Additionally, the PRQ $(=$ HR/RR) was calculated to quantify the coupling between HR and RR. The 5-min median of the baseline phase was determined for all systemic physiology data. The IQR of parameters was calculated for each individual subject.

\subsubsection{Statistical analysis}

Outliers (defined as exceeding three scaled MAD from the median) of each dataset were removed prior to the correlation analysis. The best nonlinear curve fitting (from many models including line, poly, cubic, degree 4 and 5 polynomial, piecewise linear function with 2 segments, and exponential) was estimated with R statistical software (R 3.5.2, Performance Analytics package, r-project.org) and OriginPro (version 2018b, OriginLab Corporation, Northampton, Massachusetts, USA) for each pair of parameters (8 parameters and 28 pairs) and a robust nonlinear regression was then calculated with MATLAB using the least absolute residuals method in order to avoid false-positive correlation detection. $P$-values were then obtained from goodness-offit results of each parameter pair. A false discovery rate (FDR) correction was subsequently applied to the $p$-values in order to correct for the multiple comparison situation. The bootstrapped evidence (BSE) test was conducted to find bootstrapped correlations between all parameters (28 pairs). This nonparametric method is an actual resampling procedure that takes the precision with which both the experimental $\left(H_{1}\right)$ and null $\left(H_{0}\right)$ hypothesis can be estimated into account. ${ }^{29}$ This test is also more robust compared to classical statistics by minimizing false positives while maintaining sensitivity. To investigate the dependence of cerebral parameters on seasonal changes, we applied the analysis of covariance (ANCOVA) by JASP (jasp-stats.org, version 0.9.2.0). ANCOVA is appropriate to test the main and interaction effects of categorical variables (covariates) on a continuous dependent variable. In this analysis, age and sex were selected as covariates, and a cerebral parameter and season were chosen as dependent and fixed factors, respectively. Since ANCOVA (Kruskal-Wallis nonparametric test; Dunn's post hoc comparisons; Holm correction) showed that the covariates (sex and age) have interaction effects on most cerebral variables, the effect of seasonal changes, time of day, and temperature on cerebral parameters were investigated separately for both female and male groups. For this analysis, eight subjects aged over 30 years were excluded from these evaluations in order to have a sample in a small age range (20 to 30 years of age). Finally, Cosinor analysis [Eq. (1)] and the sum of 2 cosine functions [Eq. (2)] were applied in order to find the best chronobiological fit model of the cerebral parameters:

$$
f(t)=M+A \cos \left[\frac{2 \pi(t+\varphi)}{24}\right]
$$

where $f(t)$ denotes the value of the function at time $t$ (e.g., a cerebral parameter), $M$ is the midline estimating statistic of rhythm, $A$ is the amplitude, $t$ is measured in hours, and $\varphi$ is the acrophase:

$$
f(t)=\text { base }+A_{1} \cos \left[\frac{2 \pi(t+\varphi)}{24}\right]+A_{2} \cos \left[\frac{2 \pi(t+\varphi)}{12}\right]
$$


where $f(t)$ represents the value of the function at time $t$, base is the cerebral parameter baseline value, $A_{1}$ and $A_{2}$ are the amplitudes of the cosine functions, and $\varphi$ is the acrophase.

\subsubsection{Reliability analysis}

The intrasubject variability of all data, including cerebral and cardiorespiratory parameters were analyzed by the intraclass correlation coefficient (ICC) using the R statistical software (R 3.5.2, ICC package, r-project.org). ICC is a more desirable measure of reliability, reflecting both degrees of correlation and agreement between measurements. According to Fleiss ${ }^{30}$ ICC values $<0.4$, between 0.4 and 0.6 , in the range of 0.6 and 0.75 , and $>0.75$ are indicative of poor, moderate, good, and excellent reliability, respectively.

\section{Results}

\subsection{Cerebral Oxygenation and Perfusion: Higher $\mathrm{StO}_{2}$ of Right PFC}

For $\mathrm{StO}_{2}$, we found right-dominant activity $\left(\Delta \mathrm{StO}_{2}>0\right)$, i.e., a highly significant $(p<0.0001)$ FCOA was detected in the resting state [Fig. 1(a)]. No significant $(p=0.324)$ asymmetry was found for [tHb] [Fig. 1(b)]. The intersubject mean value representing the normal value of $\mathrm{StO}_{2}$ and [tHb] was (mean $\pm \mathrm{SD}$ ) $73.0 \% \pm 5.9 \%$ and $41.4 \pm 9.3 \mu \mathrm{M}$, respectively [Figs. 1(c) and 1(d)]. For the right and left PFC the normal values of $\mathrm{StO}_{2}$ were $73.7 \% \pm 6.9 \%$ (right) and $72.3 \% \pm 6.1 \%$ (left), and of [tHb] $41.4 \pm 10.8$ (right) and $41.4 \pm 10.1$ (left) [Figs. 2(a) and 2(b)]. The absolute values of $\mathrm{StO}_{2}$ and [tHb] during the resting state are shown in Figs. 2(c) and 2(d). All data were normally distributed. From this point on, the outliers were removed and not considered for subsequent evaluations. After removal of the outliers, FCOA remained highly significant $(p<0.0001)$ [nonsignificant for $[\mathrm{tHb}](p=0.218)]$.

\subsection{Cardiorespiratory Activity}

Figures 1(e)-1(h) and 2(e), 2(h)-2(j) show the absolute values of $\mathrm{P}_{\mathrm{ET}} \mathrm{CO}_{2}, \mathrm{HR}, \mathrm{RR}$, and PRQ for the individual subjects during resting state. On the group level, mean absolute and SD values of cardiorespiratory parameters were as follows (data were normally distributed): $\mathrm{P}_{\mathrm{ET}} \mathrm{CO}_{2}$ : $39.2 \pm 4.4 \mathrm{mmHg}$, HR: $68 \pm 11$ beats/min (BPM), RR: $16.5 \pm 2.9$ breaths/min (BrPM), and PRQ: $4.2 \pm 0.9$. These values were all in the normal range for healthy adults at rest.

\subsection{Relationships with Systemic Physiology}

A correlation matrix of $\Delta \mathrm{StO}_{2}, \Delta \mathrm{tHb}, \mathrm{StO}_{2},[\mathrm{tHb}], \mathrm{P}_{\mathrm{ET}} \mathrm{CO}_{2}, \mathrm{HR}, \mathrm{RR}$, and $\log (\mathrm{PRQ})$ variables during the resting state is depicted in Fig. 3(a). In detail, Table S1 in the Supplementary Material shows curve fitted models, goodness-of-fit results, BSE parameter, and a significance level for each pair of variables. Statistically significant correlations were found between cerebral and cardiorespiratory parameters for six pairs of variables: $\Delta \mathrm{StO}_{2}$ versus RR ( $p_{\mathrm{FDR}}=0.022$, $p=0.010), \Delta \mathrm{StO}_{2}$ versus $\log (\mathrm{PRQ})\left(p_{\mathrm{FDR}}=0.024, p=0.012\right), \quad \mathrm{StO}_{2}$ versus $\mathrm{P}_{\mathrm{ET}} \mathrm{CO}_{2}$ $\left(p_{\mathrm{FDR}}<0.0001, p<0.0001\right),[\mathrm{tHb}]$ versus HR $\left(p_{\mathrm{FDR}}=0.001, p<0.0004\right),[\mathrm{tHb}]$ versus $\mathrm{RR}$ $\left(p_{\mathrm{FDR}}=0.029, p=0.016\right)$, and $[\mathrm{tHb}]$ versus $\log (\mathrm{PRQ})\left(p_{\mathrm{FDR}}<0.0001, p<0.0001\right)$.

Correlations were also observed between the cerebral parameters: $\Delta \mathrm{StO}_{2}$ versus $\Delta \mathrm{tHb}$ $\left(p_{\mathrm{FDR}}=0.008, p=0.003\right), \Delta \mathrm{StO}_{2}$ versus $\mathrm{StO}_{2}\left(p_{\mathrm{FDR}}<0.0001, p<0.0001\right), \Delta \mathrm{StO}_{2}$ versus $[\mathrm{tHb}]\left(p_{\mathrm{FDR}}=0.020, p=0.008\right)$, and $\mathrm{StO}_{2}$ versus $[\mathrm{tHb}]\left(p_{\mathrm{FDR}}<0.0001, p<0.0001\right)$. A relatively strong correlation between cardiorespiratory parameters was also present: $\mathrm{P}_{\mathrm{ET}} \mathrm{CO}_{2}$ versus $\mathrm{HR}\left(p_{\mathrm{FDR}}<0.0001, p<0.0001\right), \mathrm{P}_{\mathrm{ET}} \mathrm{CO}_{2}$ versus RR $\left(p_{\mathrm{FDR}}=0.039, p=0.022\right), \mathrm{P}_{\mathrm{ET}} \mathrm{CO}_{2}$ versus $\log (\mathrm{PRQ})\left(p_{\mathrm{FDR}}<0.0001, p<0.0001\right)$, HR versus RR ( $\left.p_{\mathrm{FDR}}=0.001, p<0.0005\right)$, HR versus $\log (\mathrm{PRQ})\left(p_{\mathrm{FDR}}<0.0001, p<0.0001\right)$, and $\mathrm{RR}$ versus $\log (\mathrm{PRQ})\left(p_{\mathrm{FDR}}<0.0001\right.$, $p<0.0001)$. Since PRQ was calculated from HR and RR, we expected a linear correlation of HR versus $\log (\mathrm{PRQ})$, and RR versus $\log (\mathrm{PRQ})$. Additionally, Fig. 3(b) illustrates the coefficient of correlation " $r$ " and BSE parameter " $\varepsilon$ " for each pair. The order of correlation starting 

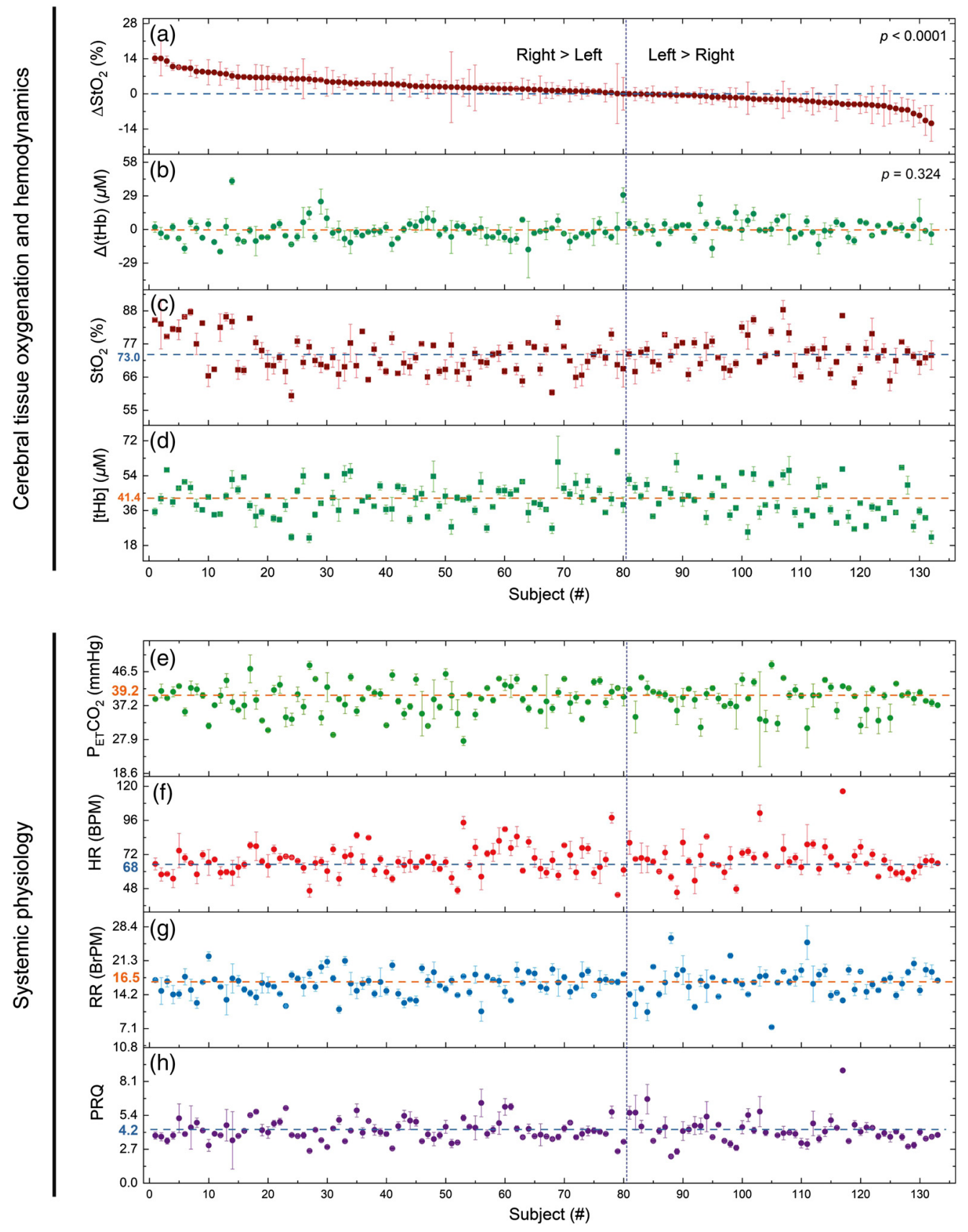

Fig. 1 (a) FCOA of $\mathrm{StO}_{2}$ at the PFC sorted in descending order on the individual subjects. (b) Asymmetry of [tHb] and absolute values of (c) $\mathrm{StO}_{2}$, (d) [tHb], (e) $\mathrm{P}_{\mathrm{ET}} \mathrm{CO}_{2}$, (f) $\mathrm{HR}$, (g) RR, and (h) PRQ displayed according to the $\Delta \mathrm{StO}_{2}$ sorting, at the PFC for individual subjects during resting state. The median and the IQR are shown for each subject.

from strongest ( $r$-value close to 1 and $\varepsilon>0.5$ ) is as follows: (1) $\mathrm{StO}_{2}$ versus $\mathrm{P}_{\mathrm{ET}} \mathrm{CO}_{2}$, (2) $\mathrm{StO}_{2}$ versus $[\mathrm{tHb}]$, (3) $\mathrm{P}_{\mathrm{ET}} \mathrm{CO}_{2}$ versus $\mathrm{HR}$, (4) [tHb] versus $\log (\mathrm{PRQ})$, (5) $\mathrm{P}_{\mathrm{ET}} \mathrm{CO}_{2}$ versus $\log (\mathrm{PRQ})$, (6) $\Delta \mathrm{StO}_{2}$ versus $\mathrm{StO}_{2}$, (7) $\mathrm{HR}$ versus $\mathrm{RR}$, and (8) [tHb] versus $\mathrm{HR}$.

\subsection{Intrasubject Variability: ICC Values Indicate Good-to-Excellent Reliability of Most Parameters}

Figure 3(c) presents the ICC values of all variables. The ICC of $\mathrm{StO}_{2},[\mathrm{tHb}], \mathrm{StO}_{2}$ (left), [tHb] (right), [tHb] (left), $\mathrm{P}_{\mathrm{ET}} \mathrm{CO}_{2}, \mathrm{HR}$, and $\mathrm{RR}$ indicates excellent reliability. The ICC of 

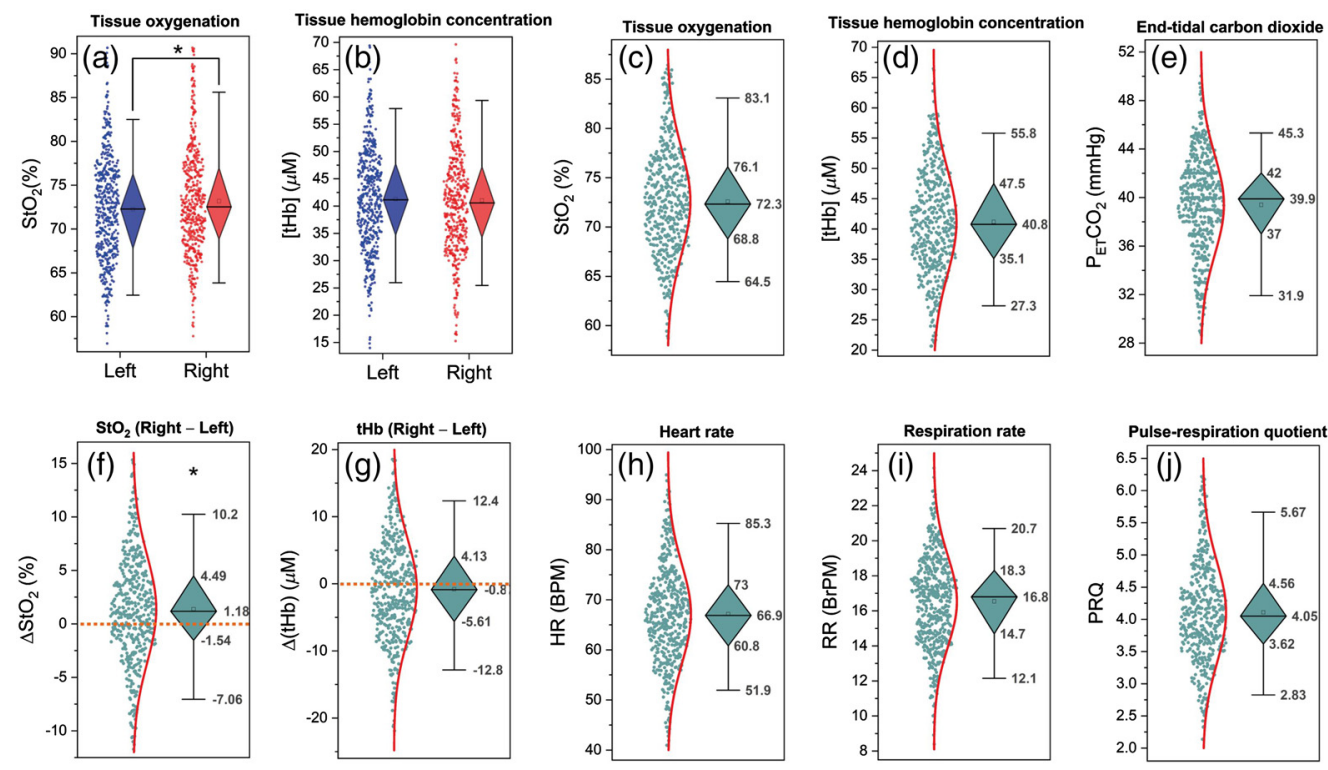

Fig. 2 Diamond box plots showing distributions of absolute (a) $\mathrm{StO}_{2}$ and (b) [tHb] values at the RPFC and L-PFC, (c) $\mathrm{StO}_{2}$, (d) [tHb], (e) $\mathrm{P}_{\mathrm{ET}} \mathrm{CO}_{2}$, (f) $\Delta \mathrm{StO}_{2}$, (g) $\Delta \mathrm{tHb}$, (h) $\mathrm{HR}$, (i) RR, and (j) PRQ values in resting state. The diamond spans the first quartile to the third quartile (IQR). A segment inside the diamond shows the median and whiskers above and below the box plots represent the $95 \%$ prediction interval. The asterisks indicate the level of high significance between absolute $\mathrm{StO}_{2}$ values of the R-PFC and L-PFC ( ${ }^{*} p<0.001$, Wilcoxon signed-rank test). Outliers are not displayed.
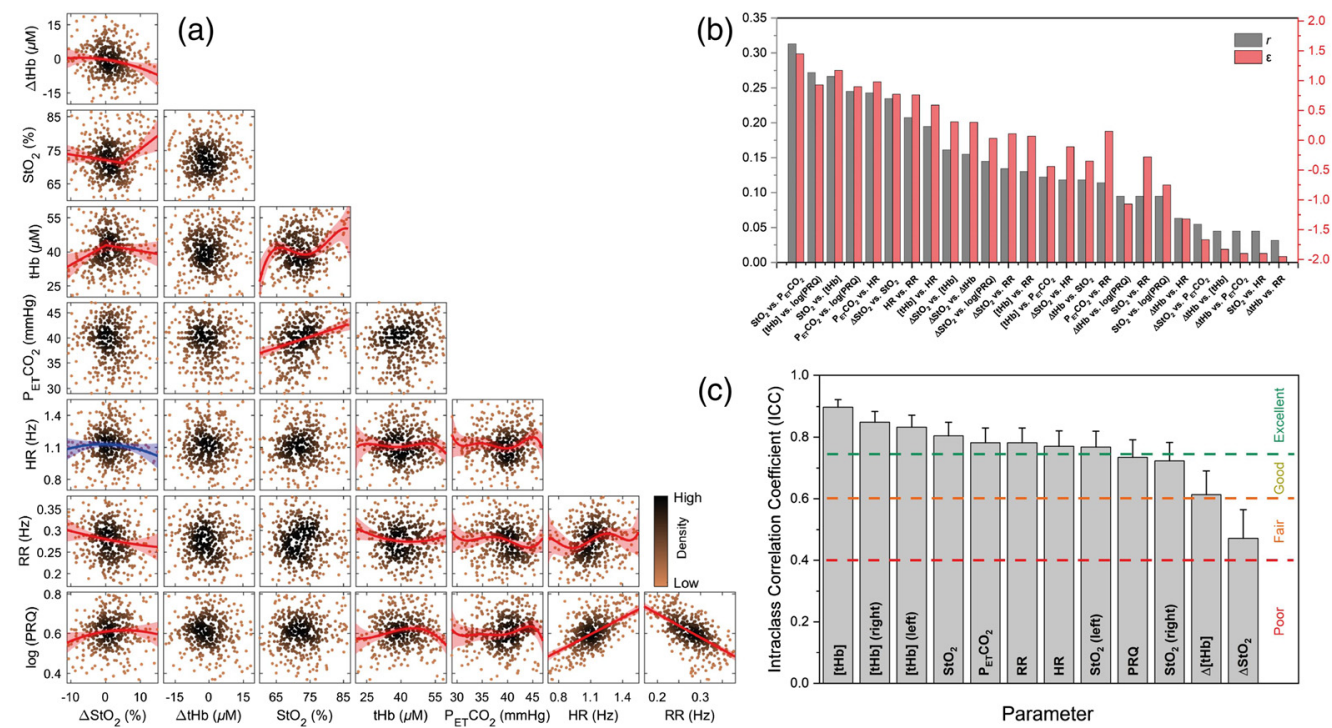

(c)

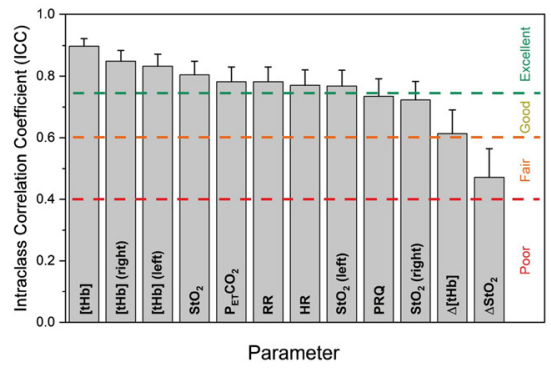

Fig. 3 (a) Correlation matrix of bivariate scatter plots of $\Delta \mathrm{StO}_{2}, \Delta \mathrm{tHb}, \mathrm{StO}{ }_{2}$, [tHb], $\mathrm{P}_{\mathrm{ET}} \mathrm{CO}_{2}, \mathrm{HR}$, $\mathrm{RR}$, and $\log (\mathrm{PRQ})$. The best nonlinear fit is presented for pairs with a significant correlation (red: significant correlation proved by both FDR-corrected and uncorrected $p$ values; blue: only uncorrected $p$ value). The level of significance is calculated from goodness-of-fit results. The red and blue shaded areas show $95 \%$ of confidence intervals. Outliers are not displayed. (b) Bar chart of $r$ and $\varepsilon$ values for each pair of $\Delta \mathrm{StO}_{2}, \Delta \mathrm{tHb}, \mathrm{StO}_{2}$, [tHb], $\mathrm{P}_{\mathrm{ET}} \mathrm{CO}_{2}, \mathrm{HR}$, RR, and $\log (\mathrm{PRQ})$ parameters (sorted in descending order of $r$-values). $\varepsilon$ values $<-0.5$, near-zero $(-0.5<\varepsilon<0.5$ ), between 0.5 and 1 , and $>1$ are indicative of no, inconclusive, moderate, and strong correlation, respectively. (c) Bar chart of ICC values for all cerebral and cardiorespiratory parameters. Error bars represent the $95 \%$ confidence interval. The reliability of the ICC is indicated. 
the PRQ, $\mathrm{StO}_{2}$ (right), and $\Delta \mathrm{tHb}$ shows good and the $\mathrm{ICC}$ of $\Delta \mathrm{StO}_{2}$ represents moderate reliability.

\subsection{Dependence of Cerebral Parameters on Sex and Seasonal Changes}

The impact of sex and seasons (infradian changes) on cerebral parameters is depicted in Fig. 4. A significant difference due to sex $(p<0.0001)$ was observed in both $\mathrm{StO}_{2}$ and [tHb]. In addition, $\Delta[\mathrm{tHb}]$ was higher for males compared to females $(p<0.001)$, but not for $\Delta \mathrm{StO}_{2}(p=0.635)$. We found that FCOA showed the same trend of seasonal changes for both female and male groups and was higher in autumn and winter compared to spring and summer (spring versus autumn, $p=0.001$; spring versus winter, $p<0.001$; summer versus autumn, $p=0.024$; summer versus winter, $p<0.001$ and autumn versus winter, $p=0.011)$. Interestingly, a Cosinor model fitted to $[\mathrm{tHb}]$ data represents completely contrary patterns for male and female groups. Absolute $[\mathrm{tHb}]$ values of males were higher in spring and summer than that of in autumn and winter. Conversely, the $[\mathrm{tHb}]$ values of females were observed at higher levels in autumn and winter in comparison with spring and summer.

\subsection{Dependence of Cerebral Parameters on Time of Day}

The effect of time of day (circadian changes) on cerebral parameters is also shown in Fig. 4. A sum of 2 cosine functions was applied to fit the circadian rhythm of the data. The same trend of higher $\mathrm{StO}_{2}$ values in the morning was observed for males and females $(p<0.001)$. Males demonstrated very high $[\mathrm{tHb}]$ values in the early morning and late evening $(p<0.001)$, but the trend was almost opposite in females $(p<0.05)$. The highest FCOA for $[\mathrm{tHb}]$ values were found at 10:00 and 14:00 for males and females, respectively. Regardless of sex, a highly significant difference was found between $\mathrm{StO}_{2}$ values of morning and afternoon $(p<0.001)$. There was also a significant difference between $\mathrm{StO}_{2}$ values of morning and evening $(p=0.011)$. No significant changes were found for [tHb] (morning versus afternoon: $p=0.061$; morning versus evening: $p=0.17$ ).

\subsection{Dependence of Cerebral Parameters on Temperature}

Figure 5 shows the changes in room temperature with respect to season $5(a)$ and time of day $5(b)$, and the temperature dependency of cerebral parameters $[5(\mathrm{c})-5(\mathrm{f})]$. The mean room temperature
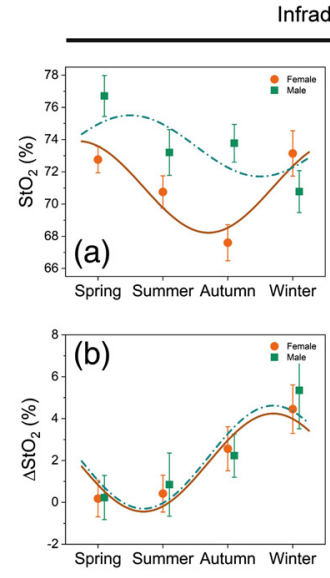
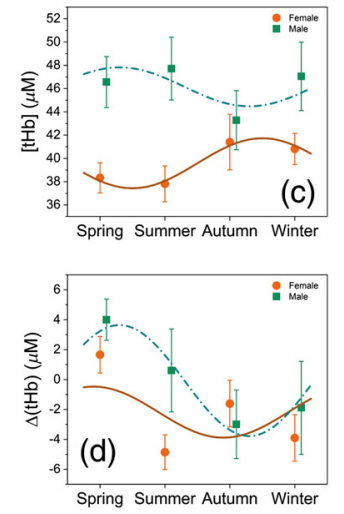
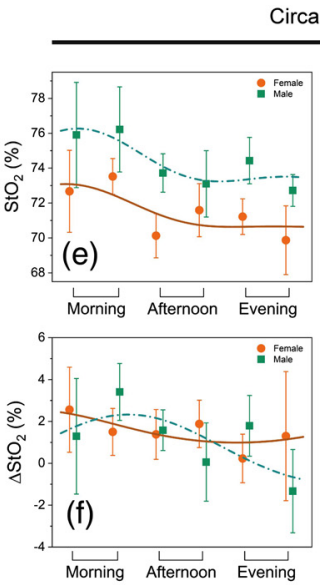

rcadian changes
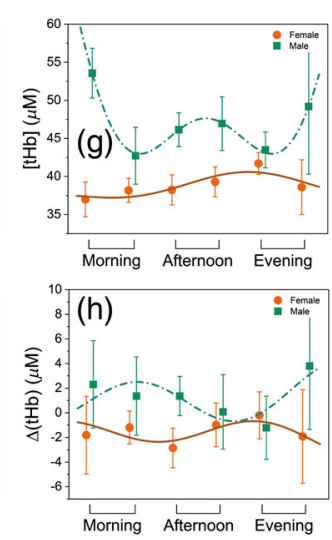

Fig. 4 Changes in $\mathrm{StO}_{2}$, [tHb], $\Delta \mathrm{StO}_{2}$, and $\Delta \mathrm{tHb}$ due to time of year [infradian changes (a)-(d)] and time of day [circadian changes (e)-(h); morning: 7:00-9:30 and 9:30-12:00; afternoon: 12:0014:30 and 14:30-17:00; evening: 17:00-19:30 and 19:30-22:00] for females (orange) and males (green). Cosinor model and the sum of 2 cosines function are fitted to infradian and circadian changes, respectively (female, dark orange lines; male, dashed green lines). Error bars represent the $95 \%$ confidence interval. 

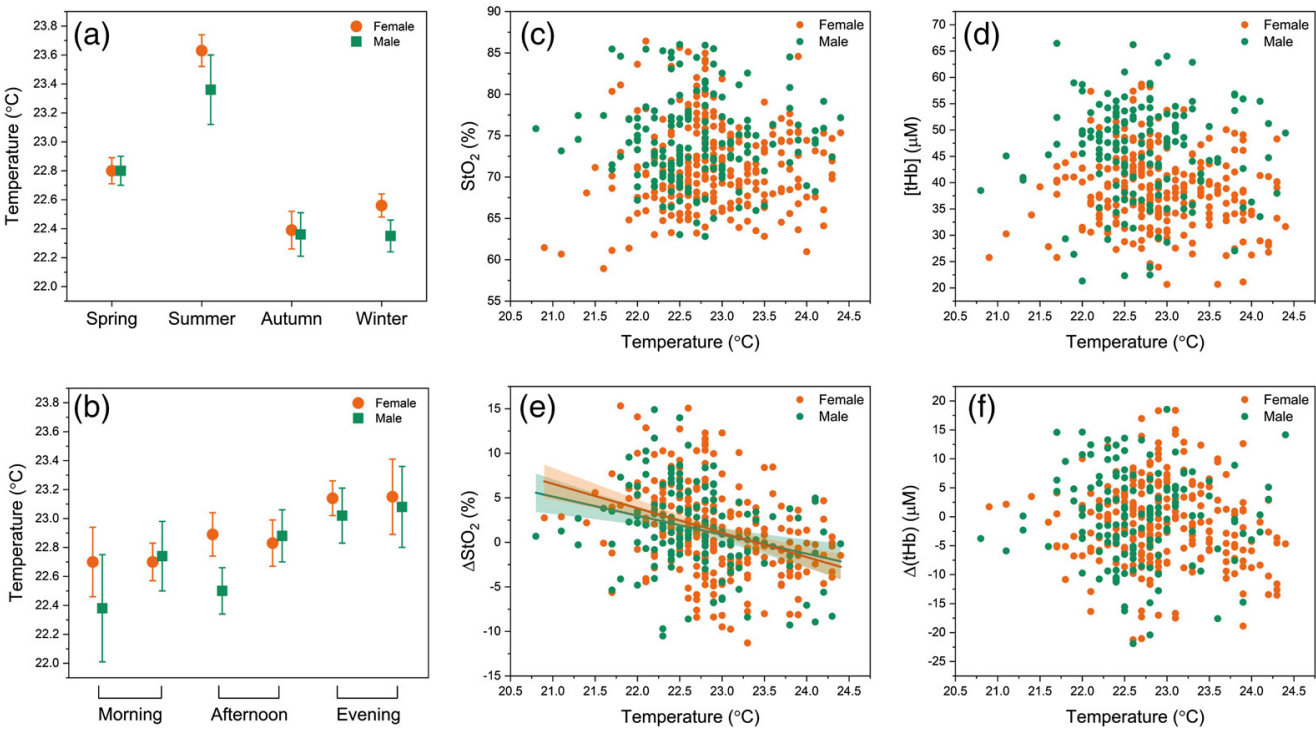

Fig. 5 Changes in temperature with (a) time of year and (b) time of day. Dependence of (c) $\mathrm{StO}_{2}$, (d) [ $\mathrm{tHb}],(\mathrm{e}) \Delta \mathrm{StO}_{2}$, and (f) $\Delta \mathrm{tHb}$ on temperature. The lines represent a linear fit and the shaded areas and error bars show $95 \%$ confidence intervals. Outliers are not displayed.

was $22.8^{\circ} \mathrm{C} \pm 0.6^{\circ} \mathrm{C}$ (range: $20.8^{\circ} \mathrm{C}$ to $24.8^{\circ} \mathrm{C}$ ). Since the room was not air-conditioned, time of day and seasonal changes had an impact on the room temperature. As expected, the maximum room temperatures were recorded in summer and during the late evening. There was no significant linear correlation between room temperature and cerebral. The only exception was $\Delta \mathrm{StO}_{2}$, which decreased with increasing room temperature (females: $r=-0.35, p<0.0001$; males: $r=-0.30, p<0.0002)$.

\subsection{Dependence of Cerebral Parameters on Mood and Chronotype}

The mean valence, arousal, and dominance ratings during resting state assessed by SAM scales (ranging from 1 to 5) was $4.02 \pm 0.69,2.92 \pm 0.91$, and $3.24 \pm 0.77$, respectively. The dependence of cerebral parameters on mood and chronotype is displayed in Fig. 6. No correlation was observed between the cerebral parameters and the positive affect scale of the PANAS
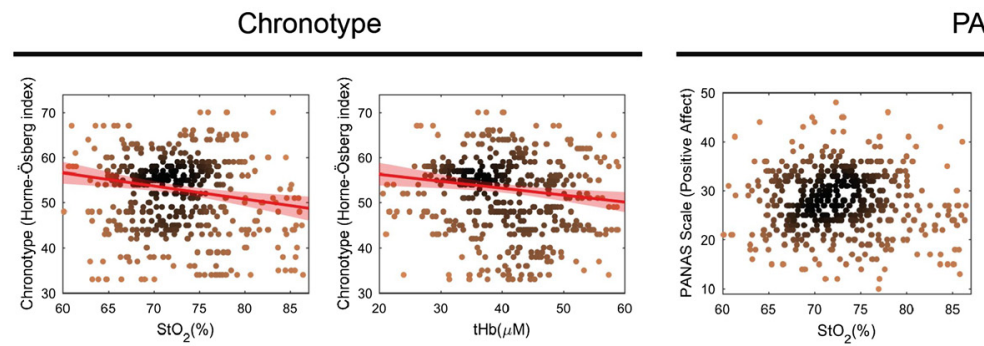

PANAS
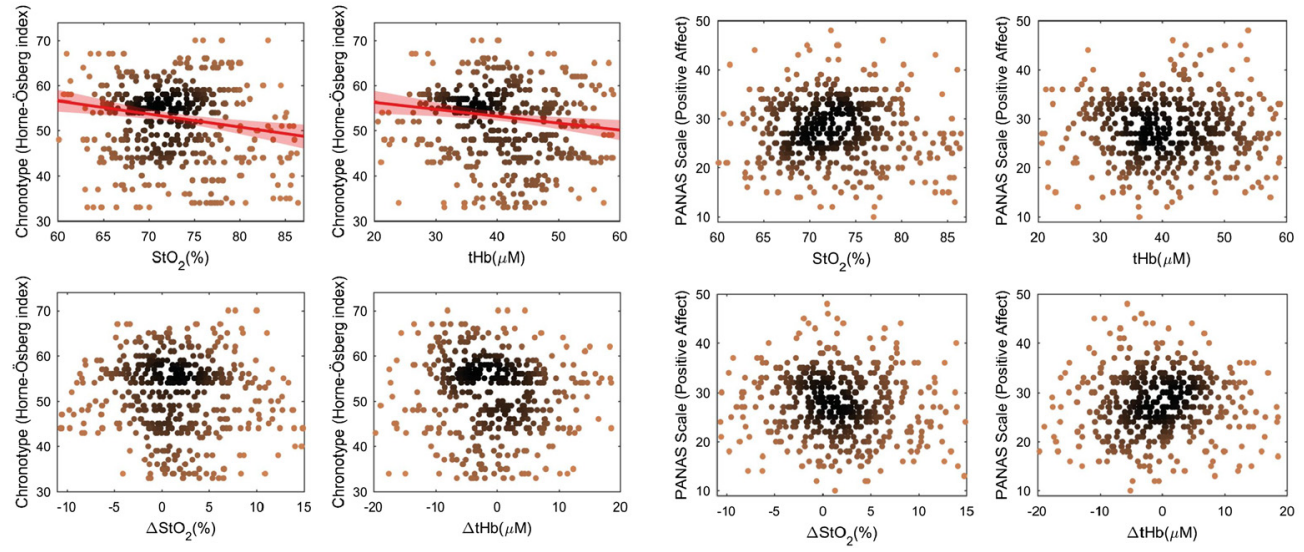

Fig. 6 The scatter plots show the dependence of $\mathrm{StO}_{2}$, [ $\left.\mathrm{tHb}\right], \Delta \mathrm{StO}_{2}$, and $\Delta \mathrm{tHb}$ on chronotype (Horne-Östberg index) and mood (positive affect scale of PANAS). Correlations between the data are indicated by a linear fit, and the red shaded areas indicate $95 \%$ confidence intervals. Outliers are not displayed. 
questionnaire. The Horne and Östberg index was calculated for each subject to measure the chronotype. The highest numbers (score: 59 to 86 ) indicate morningness and the lowest numbers (score: 16 to 41) eveningness. Scores from 42 to 58 indicate neither morningness nor eveningness. We found a linear correlation between $\mathrm{StO}_{2}$ versus chronotype $(r=-0.1, p=0.03)$, and $[\mathrm{tHb}]$ versus chronotype $(r=-0.09, p=0.04)$.

\section{Discussion}

\subsection{Absolute Values of Tissue Oxygenation and Hemoglobin Concentration}

The normal range of $\mathrm{StO}_{2}$ and [ $\mathrm{tHb}$ ] of the brain was investigated for medical applications. These absolute values are approximately in accordance with the literature. ${ }^{31,32}$ The absolute $\mathrm{StO}_{2}$ values of the PFC are in good agreement with Choi et al. (R-PFC: $74.75 \% \pm 5.83 \%$ versus. L-PFC: $75.63 \% \pm 5.86 \% ; N=30$, age: 20 to 50 years, device: Imagent, ISS Inc.). ${ }^{33}$ However, our absolute $[\mathrm{tHb}]$ values at the PFC are lower in comparison with their findings (R-PFC: $79.68 \pm 12.15 \mu \mathrm{M}$, L-PFC: $76.93 \pm 14.98 \mu \mathrm{M}$ ). Our [tHb] values are similar to those of Vernieri et al. (left versus right frontal region: $46.2 \pm 11.9 \mu \mathrm{M}$ versus $44.0 \pm 12.9 \mu \mathrm{M} ; 30$ subjects, age: $63.9 \pm 8.2$, device: Oximeter, ISS Inc.). ${ }^{34}$ Moreover, our $\mathrm{StO}_{2}$ values are a bit higher compared to our previous study $\left[\mathrm{StO}_{2}\right.$ (right) $=68.6 \%$ (IQR: $63.5 \%$ to $\left.72.4 \%\right), \mathrm{StO}_{2}$ (left) $=$ 56.8\% (IQR: $52.9 \%$ to $63.4 \%$ ); 24 subjects, age: $22.0 \pm 6.4$ years, device: OxiplexTS, ISS Inc.]. ${ }^{1}$ The reasons for the difference between studies may be the age, physiological state of subjects, or methodology.

\subsection{Absolute Values of Cardiorespiratory Parameters}

Arterial partial pressure of carbon dioxide $\left(\mathrm{PaCO}_{2}\right)$ is one of the strongest parameters that affect $\mathrm{CBF}$ and $[\mathrm{tHb}] .{ }^{19}$ Therefore, $\mathrm{PaCO}_{2}$ has been included in functional brain studies to ensure a correct interpretation of the signals. ${ }^{35-37}$ We measured $\mathrm{PaCO}_{2}$ by the $\mathrm{P}_{\mathrm{ET}} \mathrm{CO}_{2}$ method, which also provides continuous and noninvasive RR. Our $\mathrm{P}_{\mathrm{ET}} \mathrm{CO}_{2}$ values were in agreement with the literature. $^{38-41}$

Our findings showed that the mean value of HR was $68 \pm 11$ BPM (range: 41 to 116 BPM, females: $69 \pm 11 \mathrm{BPM}$, males: $66 \pm 11 \mathrm{BPM} ; p=0.036$ ). In 35,000 healthy subjects, a mean HR of 72 BPM (age: 20 and over, females: $74 \pm 0.2 \mathrm{BPM}$, males: $71 \pm 0.3 \mathrm{BPM} ; p<0.05$ ) was determined, which is close to our results. ${ }^{42}$

The mean RR value measured in our study was $16.5 \pm 2.9 \mathrm{BrPM}$, which is within typical RR for adults (range: 6.9 to $27.1 \mathrm{BrPM}$, females: $16.8 \pm 2.8 \mathrm{BPM}$, males: $16.1 \pm 3.1 \mathrm{BPM}$; $p=0.015$ ).

The PRQ is a parameter to attain the overall current state of human physiology. ${ }^{43}$ PRQ represents the state of the ANS and is a measure of cardiorespiratory coordination. PRQ is time- and sex-dependent, and changes during human development, physical activity, and body posture with specific patterns during sleep. ${ }^{43}$ The resting state PRQ distribution has a peak at $\sim 4 .{ }^{44-46}$ We also found a mean resting state PRQ of $\sim 4$ (4.2 \pm 0.9 , ranging from 2.1 to 9.0).

\subsection{Relationships with Systemic Physiology}

Although systemic physiological activity affects the absolute values of $\mathrm{StO}_{2}$ and $[\mathrm{tHb}]$, FCOA was not influenced. The reason is that both R-PFC and L-PFC are affected in the same way by systemic physiology. ${ }^{1,21}$ Although we generally confirmed this finding, we found nonlinear correlations between $\Delta \mathrm{StO}_{2}$ versus $\mathrm{RR}(p=0.022)$ and $\Delta \mathrm{StO}_{2}$ versus PRQ $(p=0.024)$.

As expected, we found a highly significant $\left(R^{2}=0.10, p<0.0001\right)$ positive linear correlation between $\mathrm{StO}_{2}$ and $\mathrm{P}_{\mathrm{ET}} \mathrm{CO}_{2}$. Our finding is in agreement with the study carried out by Miller and Mitra. ${ }^{47}$ and with the physiologically well-known $\mathrm{CO}_{2}$-response, i.e., a decrease in $\mathrm{P}_{\mathrm{ET}} \mathrm{CO}_{2}$ (hypocapnia) reduces the $\mathrm{CBF}$ by cerebral vasoconstriction. ${ }^{48}$ This reduced oxygen supply leads to a lower $\mathrm{StO}_{2} \cdot{ }^{35,36}$ Hence, $\mathrm{P}_{\mathrm{ET}} \mathrm{CO}_{2}$ is positively correlated with $\mathrm{StO}_{2}$ 


\subsection{Frontal Cortex Oxygenation Asymmetry}

In EEG studies, activity in left frontal regions is mostly associated with appetitive motivation and approach-related affect such as hope, happiness, and joy (positive affect). Conversely, the right frontal regions are related to vigilant attention and behavioral inhibition that regularly occurs during certain withdrawal-related affect such as depression and nervousness (negative affect). ${ }^{11,12,49-51}$ In general, the right frontal cortex reflects motivational systems of approach and avoidance, whereas the left frontal cortex inhibits the amygdala and downregulates negative affect. ${ }^{52,53}$ Higher right frontal activity is attributed to greater negative affect (e.g., film-induced fear and disgust), whereas positive affect (e.g., film-induced happiness) elicits a higher left frontal activity. ${ }^{54,55}$

We hypothesized that the FCOA reveals asymmetry of the PFC neuronal activity at rest. The $\mathrm{StO}_{2}$ was higher at the R-PFC than the L-PFC, indicating that the R-PFC is more activated than the L-PFC and this indicates a higher inhibitory activity or withdrawal motivation. This is reasonable considering that the subjects were in the resting state. Such rightward lateralization has also been found in the literature. ${ }^{56,57}$ Although our findings are in line with several studies indicating that the right cortices have a stronger response compared to the left ones, some studies have reported no hemispheric differences or even leftward regional lateralization. ${ }^{33,58}$ Liu et al. ${ }^{6}$ demonstrated that right or left regional laterality could be observed across different brain systems depending on multiple genetic or environmental mechanisms.

\subsubsection{FCOA as an indicator of human health}

The R-PFC plays a vital role in the brain's response to stress because this area is a primary part of both the emotion and vigilance networks. Neurons that are either the target or the releasing site of an array of stress mediators (neurotransmitter and hormone) have been recognized in this area. ${ }^{59}$ Thus FCOA is associated with specific emotional responses to mental stress and personality traits (state influence versus trait influence). ${ }^{14,52,60}$ High left frontal brain activity is more psychologically and physically healthy than relatively less left frontal brain activity. ${ }^{53,61}$ Individuals with higher L-PFC activity have lower concentrations of the stress hormone cortisol and the corticotrophin-releasing hormone, higher activity of natural killer cells, and higher antibody concentration in response to influenza vaccines. ${ }^{53,62}$ It was also demonstrated that subjects with higher L-PFC activity, recover more quickly from a negative occurrence with higher levels of psychological well-being. ${ }^{53,63}$ Conversely, dominant R-PFC activity is associated with increased activation of the hypothalamic-pituitary-adrenal axis ${ }^{59,64-66}$ and higher secretion of corticotrophin-releasing hormone and adrenal steroid hormones (e.g., glucocorticoids and adrenal androgens ). ${ }^{66-69}$ Higher R-PFC activity may occur during stressful situations, such as a test or job interview. ${ }^{70}$ An EEG study showed that FEA was shifted from the left during an easy examination session to the right during a stressful examination session. ${ }^{71}$ A higher change in FEA from the easy to the stressful session was associated with more adverse health conditions. Further research suggested that subjects with more R-PFC activity compared to L-PFC are sensitive to mental stress and prone to exhibit various stress-induced somatic disorders. ${ }^{56,64}$ Moreover, it was found that higher levels of R-PFC activation predict a reduced immune response in humans. ${ }^{59,62}$ The more an individual's FEA is changed during periods of stress, the more negative health consequences are likely to be experienced. ${ }^{49}$

We observed no correlation between cerebral parameters; in particular, FCOA and the positive affect scale of the PANAS questionnaire.

Depression is associated with an under-activation of the approach system and/or overactivation of the withdrawal system. ${ }^{72}$ Research provides support for an association between FEA and depression ${ }^{12,73,74}$ and may predict the emotional state in depression disorders. ${ }^{72}$ Although there is a small number of studies linking FEA with psychopathology, the research suggests FEA may be a promising marker of depression vulnerability. ${ }^{49}$ Decreased relative leftfrontal activity during resting state was attributed to increased vulnerability to depression. ${ }^{75}$ In adolescent boys without a history of depression, right-sided frontal activation predicted depressive symptoms 1 year later. ${ }^{76}$ Many studies indicate that FEA is a valid marker for depression vulnerability. Regardless of whether anxiety was used as a covariate or not, frontal alpha 
asymmetry indicative of relatively higher right frontal activity predicts depression, whereas the opposite is not true. ${ }^{77}$

Thus the measurement of such an FEA and FCOA may have considerable clinical value.

\subsubsection{Anatomic, physiologic, and genetic influences}

The corpus callosum provides a neuroanatomical correlation in the asymmetry of the frontal cortices. ${ }^{78}$ Negative affect generally leads to activation in the R-PFC, amygdala, inferior frontal gyrus, and insula, whereas the L-PFC may play a role in the downregulation of amygdala and is associated with reward-related cortical regions. ${ }^{53,65,79}$ The neural correlates of vigilance and sustained attention are primarily localized in the right prefrontal and parietal lobe and the thalamus. ${ }^{80}$ The link between left frontal and left amygdala activity is crucial for emotional regulation. ${ }^{73}$

Genetic models have been proposed to account for cerebral dominance, and anatomical asymmetries are likely influenced by genetic factors. However, no gene or pathway has yet been identified as a determinant of lateralization, although there are a number of candidates including LMO4, STMN4, BAI1, and IGFBP5, which were highly expressed in the right regions. ${ }^{3,81,82}$

\subsubsection{FEA as a promising marker of subject characteristics and emotions}

It was demonstrated that asymmetry in PFC neuronal activity during the resting state, measured with EEG, predicts the emotional state. ${ }^{72}$

Table 1 shows a summary of the emotions and characteristics of individuals with FEA.

\subsubsection{Environment and certain situational variable}

Experimental, environmental, and situational factors that influence approach or withdrawal motivation may affect FCOA. These variables include body posture, experimental conditions, trait

Table 1 A summary of subject characteristics and emotions with left and right dominant activity

\begin{tabular}{llc}
\hline \hline Left dominant activity & \multicolumn{1}{c}{ Right dominant activity } & References \\
\hline Anxious apprehension (e.g., worry) & Anxious arousal (e.g., panic) & 72,73 , and 83-86 \\
Maniacs & Phobias (social phobics) & 50, 83, 87 and 88 \\
Extrovert & Introvert (neuroticism) & 89 and 90 \\
Promotion (a need for growth and & Prevention (a need for safety and security) & 91 and 92 \\
advancement) & & \\
Anger, joy, and jealousy & Disgust and depression & 12 and $93-97$ \\
Hostility to social rejection & Isolation to social rejection & 60 and 98 \\
Higher socioeconomic status & Lower socioeconomic status & 99 \\
- & Defensiveness & 100 and 101 \\
- & Hopelessness & 75 and 102 \\
- & Less risk-taking & 103 \\
- & Ostracism & 104 \\
\hline- & Obsessive-compulsive disorder & 105 \\
& More concerned with making mistakes & 106 \\
\hline
\end{tabular}


variables, and timing. Thus the seasons and time of day play essential roles in FCOA scores. We found higher R-PFC activity (higher $\mathrm{StO}_{2}$ ) associated with more depression in autumn and winter compared to spring and summer. These findings are in line with a previous study. ${ }^{107}$ Indeed, it is known that the population experiences a worsening of their mood and stronger depressive symptoms (seasonal affective disorder) in winter. ${ }^{108-110}$ This is also visible in the highly significant seasonal variation in cortisol levels in winter and autumn compared to spring and summer. ${ }^{111}$ Thus it is reasonable that we found a significant influence of the seasons on FCOA.

We also found a significant dependence of the $\mathrm{StO}_{2}$ on the time of day (Fig. 4). Higher $\mathrm{StO}_{2}$ values were observed in the morning compared to the afternoon and evening. Since [tHb] was not significantly changed during the day, such an increase in $\mathrm{StO}_{2}$ may imply that more oxygenated blood was present in the brain tissue during the morning hours compared to the evening and afternoon. We can interpret this increase as reduced oxygen consumption and, thus, energy metabolism in the morning, which would be in agreement with the synaptic homeostasis hypothesis. ${ }^{112}$ This increase could also be linked to circadian effects, e.g., cortisol rhythm exerting its wake-promoting effect in the morning hours. ${ }^{113,114}$ Moreover, the present findings indicated that the time of day has no significant influence on FCOA, which is in line with the literature. ${ }^{115}$

It was also shown in this study that $\mathrm{FCOA}$ in $\mathrm{StO}_{2}$ decreased with increasing room temperature. In other words, we found that the lower the room temperature, the higher the R-PFC activation. Lower room temperature increased whole-body cooling sensation and reduced thermal comfort, especially after prolonged exposure. ${ }^{116}$ Our findings are in line with the approachmotivational model, which links higher R-PFC activation to greater withdrawal-related affect (negative affect) such as uncomfortableness, nervousness, and depression. It is known that increasing the length of daylight and temperature results in a decrease in the depression score, ${ }^{110,117}$ which indirectly confirms our findings in terms of the effects of both seasons and temperature on FCOA.

\section{Conclusion}

We found highly significant $(p<0.0001)$ FCOA, which was correlated to room temperature, RR, and PRQ but was not affected by mood or chronotype of the subject. This higher right PFC activity may be due to the more prominent inhibition activity during the resting state.

The absolute values of $\mathrm{StO}_{2}$ and [tHb] were influenced by systemic physiological activity, such as $\mathrm{P}_{\mathrm{ET}} \mathrm{CO}_{2}$, HR, RR, and PRQ, and gender.

FCOA and $\mathrm{StO}_{2}$ were dependent on season and time of day, respectively. FCOA was higher in autumn/winter compared to spring/summer, whereas $\mathrm{StO}_{2}$ was higher in the morning than in the afternoon/evening.

These relevant findings were only achievable using FD-fNIRS instrumentation that enabled the measurement of absolute values while using a SPA-fNIRS approach.

Our study demonstrates that FCOA is real, while providing unique insights to understand this remarkable phenomenon.

\section{Disclosures}

The authors declared no potential conflicts of interest with respect to the research, authorship, and/or publication of this article.

\section{Acknowledgments}

We would like to thank our students for their work and our subjects for participating in this study. The financial support of the Software AG Foundation (Grant No. P12117) and the Christophorus Foundation (Grant Nos. 253CST and 355CST) is gratefully acknowledged. We thank Oliver Kress, PhD for valuable comments and proofreading of the manuscript. 


\section{References}

1. F. Scholkmann, H. Zohdi, and U. Wolf, "Right-left asymmetry of prefrontal cerebral oxygenation: does it depend on systemic physiological activity, absolute tissue oxygenation or hemoglobin concentration?" Adv. Exp. Med. Biol. 1232, 105-112 (2020).

2. A. Hougaard et al., "Cerebral asymmetry of fMRI-BOLD responses to visual stimulation," PLoS One 10, e0126477 (2015).

3. T. Sun and C. A. Walsh, "Molecular approaches to brain asymmetry and handedness," Nat. Rev. Neurosci. 7, 655-662 (2006).

4. K. Hugdahl, "Symmetry and asymmetry in the human brain," Eur. Rev. 13, 119-133 (2005).

5. F. Homae, "A brain of two halves: insights into interhemispheric organization provided by near-infrared spectroscopy," Neuroimage 85, 354-362 (2014).

6. H. Liu et al., "Evidence from intrinsic activity that asymmetry of the human brain is controlled by multiple factors," Proc. Natl. Acad. Sci. U. S. A. 106, 20499-20503 (2009).

7. P. K. Mutha, K. Y. Haaland, and R. L. Sainburg, "The effects of brain lateralization on motor control and adaptation," J. Mot. Behav. 44, 455-469 (2012).

8. Y. Wang et al., "Multimodal mapping of the face connectome," Nat. Hum. Behav. 4, 397-411 (2020).

9. R. J. Davidson, "Emotion and affective style: hemispheric substrates," Psychol. Sci. 3, 39-43 (1992).

10. R. J. Davidson, "Affective style and affective disorders: perspectives from affective neuroscience," Cognit. Emot. 12, 307-330 (1998).

11. P. A. Gable, L. B. Neal, and A. H. Threadgill, "Regulatory behavior and frontal activity: considering the role of revised-BIS in relative right frontal asymmetry," Psychophysiology 55, e12910 (2018).

12. B. D. Nelson et al., "Depression symptom dimensions and asymmetrical frontal cortical activity while anticipating reward," Psychophysiology 55, e12892 (2018).

13. P. C. Schmid et al., "Frontal cortical effects on feedback processing and reinforcement learning: relation of EEG asymmetry with the feedback-related negativity and behavior," Psychophysiology 55, e12911 (2018).

14. W. Ishikawa et al., "New method of analyzing NIRS data from prefrontal cortex at rest," Adv. Exp. Med. Biol. 789, 391-397 (2013).

15. P. Pinti et al., "The present and future use of functional near-infrared spectroscopy (fNIRS) for cognitive neuroscience," Ann. N. Y. Acad. Sci. 1464(1), 5-29 (201820).

16. H. Zohdi et al., "Long-term changes in optical properties ( $\mu \mathrm{a}, \mu$ 's, $\mu$ eff and DPF) of human head tissue during functional neuroimaging experiments," Adv. Exp. Med. Biol. 1072, 331-337 (2018).

17. F. Scholkmann et al., "Absolute values of optical properties ( $\mu \mathrm{a}, \mu$ 's, $\mu$ eff and DPF) of human head tissue: dependence on head region and individual," Adv. Exp. Med. Biol. 1072, 325-330 (2018).

18. F. Lange and I. Tachtsidis, "Clinical brain monitoring with time domain NIRS: a review and future perspectives," Appl. Sci. 9, 1612 (2019).

19. F. Scholkmann et al., "A review on continuous wave functional near-infrared spectroscopy and imaging instrumentation and methodology," Neuroimage 85, 6-27 (2014).

20. F. Scholkmann et al., "Effect of short-term colored-light exposure on cerebral hemodynamics and oxygenation, and systemic physiological activity," Neurophotonics 4, 045005 (2017).

21. N. Nasseri et al., "Impact of changes in systemic physiology on fNIRS/NIRS signals: analysis based on oblique subspace projections decomposition," Adv. Exp. Med. Biol. 1072, 119-125 (2018).

22. H. Zohdi, F. Scholkmann, and U. Wolf, "Long-term blue light exposure changes frontal and occipital cerebral hemodynamics: not all subjects react the same," Adv. Exp. Med. Biol. 1269 (2020).

23. R. C. Oldfield, "The assessment and analysis of handedness: the Edinburgh inventory," Neuropsychologia 9, 97-113 (1971). 
24. D. Watson, L. C. Anna, and A. Tellegen, "Development and validation of brief measures of positive and negative affect: the PANAS scales," J. Pers. Soc. Psychol. 54, 1063 (1988).

25. M. M. Bradley and P. J. Lang, "Measuring emotion: the self-assessment manikin and the semantic differential," J. Behav. Ther. Exp. Psychiatry 25, 49-59 (1994).

26. J. A. Horne and O. Östberg, "A self-assessment questionnaire to determine morningnesseveningness in human circadian rhythms," Int. J. Chronobiol. 4(2), 97-110 (1976).

27. H. H. Jasper, "The ten-twenty electrode system of the International Federation," Electroencephalogr. Clin. Neurophysiol. 10, 370-375 (1958).

28. F. Scholkmann et al., "How to detect and reduce movement artifacts in near-infrared imaging using moving standard deviation and spline interpolation," Physiol. Meas. 31, 649-662 (2010).

29. D. S. Schwarzkopf, "Non-dichotomous inference using bootstrapped evidence," bioRxiv, p. 017327 (2015).

30. J. Fleiss, The Design and Analysis of Clinical Experiments, Wiley, New York (1986).

31. V. Quaresima et al., "Bilateral prefrontal cortex oxygenation responses to a verbal fluency task: a multichannel time-resolved near-infrared topography study," J. Biomed. Opt. 10, 011012 (2005).

32. Y. Murayama et al., "Relation between cognitive function and baseline concentrations of hemoglobin in prefrontal cortex of elderly people measured by time-resolved near-infrared spectroscopy," Adv. Exp. Med. Biol. 977, 269-276 (2017).

33. J. Choi et al., "Noninvasive determination of the optical properties of adult brain: nearinfrared spectroscopy approach," J. Biomed. Opt. 9(1), 221-229 (2004).

34. F. Vernieri et al., "Transcranial Doppler and near-infrared spectroscopy can evaluate the hemodynamic effect of carotid artery occlusion," Stroke 35, 64-70 (2004).

35. F. Scholkmann et al., "Cerebral hemodynamic and oxygenation changes induced by inner and heard speech: a study combining functional near-infrared spectroscopy and capnography," J. Biomed. Opt. 19, 017002 (2014).

36. F. Scholkmann et al., "End-tidal $\mathrm{CO}_{2}$ : an important parameter for a correct interpretation in functional brain studies using speech tasks," Neuroimage 66, 71-79 (2013).

37. F. Scholkmann, M. Wolf, and U. Wolf, "The effect of inner speech on arterial $\mathrm{CO}_{2}$ and cerebral hemodynamics and oxygenation: a functional NIRS study," in Oxygen Transport to Tissue XXXV, S. Van Huffel et al., Eds., pp. 81-87, Springer, New York (2013).

38. C. J. Gauthier et al., "Absolute quantification of resting oxygen metabolism and metabolic reactivity during functional activation using QUO2 MRI," Neuroimage 63, 1353-1363 (2012).

39. J. Hinkelbein et al., "Accuracy and precision of three different methods to determine $\mathrm{Pco}_{2}$ $\left(\mathrm{P}_{\mathrm{a}} \mathrm{CO}_{2}\right.$ vs. $\mathrm{P}_{\mathrm{et}} \mathrm{CO}_{2}$ vs. $\left.P_{\mathrm{tc}} \mathrm{CO}_{2}\right)$ during interhospital ground transport of critically ill and ventilated adults," J. Trauma 65, 10-18 (2008).

40. A. M. Golestani et al., "Mapping the end-tidal $\mathrm{CO}_{2}$ response function in the resting-state BOLD fMRI signal: spatial specificity, test-retest reliability and effect of fMRI sampling rate," Neuroimage 104, 266-277 (2015).

41. I. Lajoie, F. B. Tancredi, and R. D. Hoge, "Regional reproducibility of BOLD calibration parameter $\mathrm{M}, \mathrm{OEF}$ and resting-state $\mathrm{CMRO}_{2}$ measurements with QUO2 MRI," PLoS One 11, e0163071-31 (2016).

42. Y. Ostchega et al., "Resting pulse rate reference data for children, adolescents, and adults: United States, 1999-2008," Natl. Health Stat. Rep. 41, 1-16 (2011).

43. F. Scholkmann and U. Wolf, "The pulse-respiration quotient: a powerful but untapped parameter for modern studies about human physiology and pathophysiology," Front. Physiol. 10, 1-18 (2019).

44. F. Scholkmann, H. Zohdi, and U. Wolf, "The resting-state pulse-respiration quotient of humans: lognormally distributed and centred around a value of four," Physiol. Res. 68, 1027-1032 (2019).

45. M. Moser et al., "Phase and frequency coordination of cardiac and respiratory function," Biol. Rhythm Res. 26, 100-111 (1995).

46. D. von Bonin et al., "Adaption of cardio-respiratory balance during day-rest compared to deep sleep-An indicator for quality of life?” Psychiatry Res 219, 638-644 (2014). 
47. S. Miller and K. Mitra, "NIRS-based cerebrovascular regulation assessment: exercise and cerebrovascular reactivity," Neurophotonics 4, 041503 (2017).

48. K. Szabo et al., "Hypocapnia induced vasoconstriction significantly inhibits the neurovascular coupling in humans," J. Neurol. Sci. 309, 58-62 (2011).

49. S. J. Reznik and J. J. B. Allen, "Frontal asymmetry as a mediator and moderator of emotion: an updated review," Psychophysiology 55, e12965 (2018).

50. R. J. Davidson, D. C. Jackson, and N. H. Kalin, "Emotion, plasticity, context, and regulation: perspectives from affective neuroscience," Psychol. Bull. 126, 890-909 (2000).

51. H. Fischer et al., "Right-sided human prefrontal brain activation during acquisition of conditioned fear," Emotion 2, 233-241 (2002).

52. J. J. B. Allen et al., "Frontal EEG alpha asymmetry and emotion: from neural underpinnings and methodological considerations to psychopathology and social cognition," Psychophysiology 55, e13028 (2018).

53. R. J. Davidson, "What does the prefrontal cortex 'do' in affect: perspectives on frontal EEG asymmetry research," Biol. Psychol. 67, 219-234 (2004).

54. A. J. Tomarken, R. J. Davidson, and J. B. Henriques, "Resting frontal brain asymmetry predicts affective responses to films," J. Pers. Soc. Psychol. 59, 791-801 (1990).

55. R. E. Wheeler, R. J. Davidson, and A. J. Tomarken, "Frontal brain asymmetry and emotional reactivity: a biological substrate of affective style," Psychophysiology 30, 82-89 (1993).

56. W. Ishikawa et al., "Correlation between asymmetry of spontaneous oscillation of hemodynamic changes in the prefrontal cortex and anxiety levels: a near-infrared spectroscopy study," J. Biomed. Opt. 19, 027005 (2014).

57. Y. Murayama, L. Hu, and K. Sakatani, "Relation between prefrontal cortex activity and respiratory rate during mental stress tasks: a near-infrared spectroscopic study," Adv. Exp. Med. Biol. 923, 209-214 (2016).

58. A. J. Metz et al., "Continuous coloured light altered human brain haemodynamics and oxygenation assessed by systemic physiology augmented functional near-infrared spectroscopy," Sci. Rep. 7, 10027 (2017).

59. J. Wang et al., "Perfusion functional MRI reveals cerebral blood flow pattern under psychological stress," Proc. Natl. Acad. Sci. U. S. A. 102, 17804-17809 (2005).

60. E. Harmon-Jones and P. A. Gable, "On the role of asymmetric frontal cortical activity in approach and withdrawal motivation: an updated review of the evidence," Psychophysiology 55, e12879 (2018).

61. E. Harmon-Jones, "Contributions from research on anger and cognitive dissonance to understanding the motivational functions of asymmetrical frontal brain activity," Biol. Psychol. 67, 51-76 (2004).

62. M. A. Rosenkranz et al., "Affective style and in vivo immune response: neurobehavioral mechanisms," Proc. Natl. Acad. Sci. U. S. A. 100, 11148-11152 (2003).

63. D. C. Jackson et al., "Now you feel it, now you don't: frontal brain electrical asymmetry and individual differences in emotion regulation," Psychol. Sci. 14, 612-617 (2003).

64. K. Sakatani, "Optical diagnosis of mental stress: review," Adv. Exp. Med. Biol. 737, 89-95 (2012).

65. R. M. Buijs and C. G. Van Eden, "The integration of stress by the hypothalamus, amygdala and prefrontal cortex: balance between the autonomic nervous system and the neuroendocrine system," Prog. Brain Res. 126, 117-132 (2000).

66. M. Tanida, M. Katsuyama, and K. Sakatani, "Relation between mental stress-induced prefrontal cortex activity and skin conditions: a near-infrared spectroscopy study," Brain Res. 1184, 210-216 (2007).

67. K. E. Habib, P. W. Gold, and G. P. Chrousos, "Neuroendocrinology of stress," Endocrinol. Metab. Clin. 30, 695-728 (2001).

68. C. Tsigos and G. P. Chrousos, "Hypothalamic-pituitary-adrenal axis, neuroendocrine factors and stress," J. Psychosom. Res. 53, 865-871 (2002).

69. M. Tanida, M. Katsuyama, and K. Sakatani, "Effects of fragrance administration on stressinduced prefrontal cortex activity and sebum secretion in the facial skin," Neurosci. Lett. 432, 157-161 (2008). 
70. B. S. McEwen and E. N. Lasley, The End of Stress as We Know It, Joseph Henry Press, Washington, D.C. (2002).

71. R. S. Lewis, N. Y. Weekes, and T. H. Wang, "The effect of a naturalistic stressor on frontal EEG asymmetry, stress, and health," Biol. Psychol. 75, 239-247 (2007).

72. A. H. Kemp et al., "Disorder specificity despite comorbidity: resting EEG alpha asymmetry in major depressive disorder and post-traumatic stress disorder," Biol. Psychol. 85, 350-354 (2010).

73. G. E. Bruder, J. W. Stewart, and P. J. McGrath, "Right brain, left brain in depressive disorders: clinical and theoretical implications of behavioral, electrophysiological and neuroimaging findings," Neurosci. Biobehav. Rev. 78, 178-191 (2017).

74. N. van der Vinne et al., "Frontal alpha asymmetry as a diagnostic marker in depression: fact or fiction? A meta-analysis," NeuroImage Clin. 16, 79-87 (2017).

75. R. Nusslock et al., "Cognitive vulnerability and frontal brain asymmetry: common predictors of first prospective depressive episode," J. Abnorm. Psychol. 120, 497-503 (2011).

76. A. M. Mitchell and P. Pössel, "Frontal brain activity pattern predicts depression in adolescent boys," Biol. Psychol. 89, 525-527 (2012).

77. P. Pössel et al., "A longitudinal study of cortical EEG activity in adolescents," Biol. Psychol. 78, 173-178 (2008).

78. D. J. L. G. Schutter and E. Harmon-Jones, "The corpus callosum: a commissural road to anger and aggression," Neurosci. Biobehav. Rev. 37, 2481-2488 (2013).

79. R. J. Davidson, "Darwin and the neural bases of emotion and affective style," Ann. N. Y. Acad. Sci. 1000, 316-336 (2003).

80. M. Sarter, B. Givens, and J. P. Bruno, "The cognitive neuroscience of sustained attention: where top-down meets bottom-up," Brain Res. Rev. 35, 146-160 (2001).

81. T. Sun et al., "Early asymmetry of gene transcription in embryonic human left and right cerebral cortex," Science 308, 1794-1798 (2005).

82. C. Lai et al., "A forkhead-domain gene is mutated in a severe speech and language disorder," Nature 413, 519-523 (2001).

83. R. J. Davidson et al., "While a phobic waits: regional brain electrical and autonomic activity in social phobics during anticipation of public speaking," Biol. Psychiatry 47, 85-95 (2000).

84. G. Wiedemann et al., "Frontal brain hipoactivity as a biological substrate of anxiety in patients with panic disorders," Arch. Gen. Psychiatry 56, 78-84 (1999).

85. W. Heller et al., "Patterns of regional brain activity differentiate types of anxiety," J. Abnorm. Psychol. 106, 376-385 (1997).

86. E. E. Smith, L. Zambrano-Vazquez, and J. J. B. Allen, "Patterns of alpha asymmetry in those with elevated worry, trait anxiety, and obsessive-compulsive symptoms: a test of the worry and avoidance models of alpha asymmetry," Neuropsychologia 85, 118-126 (2016).

87. K. Kano et al., "The topographical features of EEGs in patients with affective disorders Kunio," Electroencephalogr. Clin. Neurophysiol. 83, 124-129 (1992).

88. D. A. Moscovitch et al., "Frontal EEG asymmetry and symptom response to cognitive behavioral therapy in patients with social anxiety disorder," Biol. Psychol. 87, 379-385 (2011).

89. T. Canli et al., "An fMRI study of personality influences on brain reactivity to emotional stimuli," Behav. Neurosci. 115, 33-42 (2001).

90. C. L. Smith and M. A. Bell, "Stability in infant frontal asymmetry as a predictor of toddlerhood internalizing and externalizing behaviors," Dev. Psychobiol. 52, 158-167 (2010).

91. D. C. Molden, Y. L. Angela, and E. T. Higgins, "Motivations for promotion and prevention," in Handbook of Motivation Science, J. Shah and W. Gardner, Eds., pp. 169-187, Guilford Press, New York (2008).

92. D. M. Amodio et al., "Implicit regulatory focus associated with asymmetrical frontal cortical activity," J. Exp. Soc. Psychol. 40, 225-232 (2004).

93. E. Harmon-Jones, C. K. Peterson, and C. R. Harris, "Jealousy: novel methods and neural correlates," Emotion 9, 113-117 (2009).

94. N. J. Kelley et al., "Jealousy increased by induced relative left frontal cortical activity," Emotion 15, 550-555 (2015). 
95. N. A. Jones and N. A. Fox, "Electroencephalogram asymmetry during emotionally evocative films and its relation to positive and negative affectivity," Brain Cognit. 20, 280-299 (1992).

96. L. A. Killeen and D. M. Teti, "Mothers' frontal EEG asymmetry in response to infant emotion states and mother-infant emotional availability, emotional experience, and internalizing symptoms," Dev. Psychopathol. 24, 9-21 (2012).

97. C. S. Carver and E. Harmon-Jones, "Anger is an approach-related affect: evidence and implications," Psychol. Bull. 135, 183-204 (2009).

98. J. E. Beeney et al., "EEG asymmetry in borderline personality disorder and depression following rejection," Pers. Disord. Theory Res. Treat. 5, 178-185 (2014).

99. A. J. Tomarken et al., "Resting frontal brain activity: linkages to maternal depression and socio-economic status among adolescents," Biol. Psychol. 67, 77-102 (2004).

100. J. P. Kline and S. Allen, "The failed repressor: EEG asymmetry as a moderator of the relation between defensiveness and depressive symptoms," Int. J. Psychophysiol. 68, 228-234 (2008).

101. N. W. Crost, C. A. Pauls, and J. Wacker, "Defensiveness and anxiety predict frontal EEG asymmetry only in specific situational contexts," Biol. Psychol. 78, 43-52 (2008).

102. J. A. Coan and J. J. B. Allen, "Frontal EEG asymmetry as a moderator and mediator of emotion," Biol. Psychol. 67, 7-50 (2004).

103. L. R. R. Gianotti et al., "Tonic activity level in the right prefrontal cortex predicts individuals' risk taking," Psychol. Sci. 20, 33-38 (2009).

104. T. Kawamoto, H. Nittono, and M. Ura, "Cognitive, affective, and motivational changes during ostracism: an ERP, EMG, and EEG study using a computerized cyberball task," Neurosci. J. 2013, 1-11 (2013).

105. M. Ischebeck et al., "Altered frontal EEG asymmetry in obsessive-compulsive disorder," Psychophysiology 51, 596-601 (2014).

106. K. Nash, M. Inzlicht, and I. McGregor, "Approach-related left prefrontal EEG asymmetry predicts muted error-related negativity," Biol. Psychol. 91, 96-102 (2012).

107. C. K. Peterson and E. Harmon-Jones, "Circadian and seasonal variability of resting frontal EEG asymmetry," Biol. Psychol. 80, 315-320 (2009).

108. L. Päeske et al., EEG Functional Connectivity Detects Seasonal Changes, Springer, Singapore (2019).

109. D. Armbruster, B. Brocke, and A. Strobel, "Winter is coming: seasonality and the acoustic startle reflex," Physiol. Behav. 169, 178-183 (2017).

110. A. Magnusson, "An overview of epidemiological studies on seasonal affective disorder," Acta Psychiatr. Scand. 101, 176-184 (2000).

111. J. A. King et al., "Sequence and seasonal effects of salivary cortisol," Behav. Med. 26, 67-73 (2000).

112. G. Tononi and C. Cirelli, "Sleep function and synaptic homeostasis," Sleep Med. Rev. 10, 49-62 (2006).

113. C. Cajochen, S. Chellappa, and C. Schmidt, "What keeps us awake?- -the role of clocks and hourglasses, light, and melatonin," Int. Rev. Neurobiol. 93, 57-90 (2010).

114. A. J. Metz et al., "Brain tissue oxygen saturation increases during the night in adolescents," in Oxygen Transport to Tissue XXXV, S. Van Huffel et al., Eds., pp. 113-119, Springer, New York (2013).

115. J. R. Velo et al., "Should it matter when we record? Time of year and time of day as factors influencing frontal EEG asymmetry," Biol. Psychol. 91, 283-291 (2012).

116. K. W. Tham and H. C. Willem, "Room air temperature affects occupants' physiology, perceptions and mental alertness," Build. Environ. 45, 40-44 (2010).

117. J. Molin et al., "The influence of climate on development of winter depression," J. Affect. Disord. 37, 151-155 (1996).

Hamoon Zohdi received his MSc degree in biomedical engineering from the University of Bern in 2016. He is a PhD student at the Institute of Complementary and Integrative Medicine, University of Bern, Bern, Switzerland. His current research focuses on fNIRS with a special 
interest in investigating changes in cerebral and systemic physiology in humans elicited by different colored light exposures.

Felix Scholkmann received his PhD from the University of Zürich, Zürich, Switzerland, in 2014. He is a research associate at the University of Bern and a postdoc and a research associate at the University of Zurich. His research focuses on biomedical signal processing, biophotonics/ neurophotonics (development and application of NIRS), neuroscience, integrative physiology, and biophysics.

Ursula Wolf is a full professor at the Medical Faculty of the University of Bern and the director of the Institute of Complementary and Integrative Medicine. Her research interests focus on integrative medicine, in particular patient centered research, integrative physiology, and clinical studies. 